\title{
Proximisation discursive et co-construction de communauté sur Twitch
}

\author{
Elise Choquet ${ }^{1, *}$ et Natalia Marcela Osorio Ruiz ${ }^{1}$ \\ 1Laboratoire LHUMAIN, Université Paul Valéry Montpellier 3 Route de Mende 34199 Montpellier Cedex 5
}

\begin{abstract}
Résumé. De nos jours, de multiples dispositifs et plateformes numériques offrent aux internautes l'opportunité de dépasser les contraintes de co-présence dans l'espace physique et de se rapprocher les uns des autres en fonctions de leurs points d'intérêts et affinités. Néanmoins, au sein de ces interactions numériques, les enjeux sont différents selon le type de participant. Dans le cas des plateformes de diffusion de sessions de jeux vidéo comme Twitch, pour l'instance de production représentée par le joueur, l'enjeu ne se limite pas à la production de contenu. En effet, le joueur doit animer sa diffusion afin d'offrir non seulement du contenu vidéoludique mais également des échanges interactionnels aux spectateurs. Bien que le partage d'une passion commune amène les divers acteurs à se réunir autour de ces sessions, il existe un enjeu économique qui dépend des abonnements des spectateurs à la chaîne du joueur. Dès lors, en tant que pierre angulaire de cette pratique, le joueur déploie des stratégies pour tisser un lien de confiance avec ses spectateurs. Dans la présente étude, nous nous concentrons sur les procédures discursives qui lui permettent de co-construire et faire perdurer le lien social à travers une communauté en ligne dans le cadre d'une pratique vidéoludique.
\end{abstract}

Mots-clés : Proximité, interaction, jeux vidéo

\begin{abstract}
Discursive "proximisation" and community coconstruction in Twitch. Actually, multiple devices and digital platforms give to internet users the opportunity to reach copresence constraints in physical space and to get closer to each other in order to its points of interest and affinities. Nevertheless, inside these digital interactions the issues are different according to the type of participant. In the case of video game session delivery platforms as Twitch, for the production instance represented by the gamer, the issue is not limited to the production of content. Indeed, the gamer have to animate its diffusion in order to offer not only video game content but also interactive exchanges to spectators. Although sharing a common passion brings different actors to gather around game sessions, there is an economic issue that depends of spectator subscription to the player's channel. Consequently, as a cornerstone of this practice the gamer deploys strategies for closer a trust link with his spectators. In this study, we are going to focus on discursive procedures that allows him to co-build and perpetuate the social link through an online community as part of a video game practice.
\end{abstract}

Keywords : Proximity, interaction, videogame

\footnotetext{
*Corresponding author : elise.choquet@live.fr
} 


\section{Introduction}

Messageries de courrier électronique ou instantanées, espaces de clavardages, les forums, etc. ne cessent de questionner le rapport au langage, pris dans la triade des nouvelles cultures digitales où s'hybrident écrit, oral et interactivité. De fait les discours numériques inhérents à ces pratiques donnent à observer une évolution de la langue française au sein des espaces publics : c'est assurément le cas des interactions ludiques en ligne. Le présent article est consacré plus spécifiquement aux pratiques interactionnelles déployées au sein de la plateforme numérique Twitch. L'objet vise plus précisément à identifier les procédures discursives et praxéologiques de proximisation (selon la perspective de Fastrez et Meyer, 1999) effectuées par le joueur, en tant qu'instance de production (Charaudeau, 2011), mais aussi par les spectateurs dans la dynamique d'une co-construction de communauté. La prise en compte de deux acteurs principaux se justifie par la nature propre de l'interaction où la présence et les énoncés d'autrui justifient les procédures mises en place. S'atteler par ailleurs à rendre compte des fonctionnements discursifs à l'aune de ce double ancrage thématique (notions de proximité et de communauté), luimême au cœur des pratiques numériques, nous paraît constituer un défi pour tout linguiste s'efforçant de saisir qualitativement les évolutions contemporaines de la langue dans ses usages ordinaires massifs.

Nous commencerons par présenter l'ancrage théorique de notre recherche, qui sera suivi d'une présentation du terrain d'étude et de la méthodologie de récolte et d'analyse des données. Une étude à titre d'illustration sera présentée in fine sur deux extraits du corpus issus de la même session de jeu.

\section{Twitch}

Les données présentées sont issues de Twitch, une plateforme de diffusion de sessions créatives (peinture, cuisine, etc.), de jeux vidéo et d'émissions en direct. Initialement, Twitch n'était que la partie dédiée aux jeux de Justin.tv, plateforme consacrée aux médias créée en 2007. Cette dernière ferme ses portes en 2014 après que ses créateurs ont décidé de recentrer le site sur le contenu lié aux jeux vidéo: Twitch devient alors une entité propre dont le succès ne cesse de croître. Par exemple, pour la seule année 2015, ce sont près de 17500 messages par minute, et plus de 400000 années de vidéos produites d'après la rétrospective officielle de l'année. À l'heure actuelle, Twitch domine le marché des vidéos en direct ${ }^{\mathrm{i}}$. D'importance mondiale, la plateforme permet aux joueurs et spectateurs du monde entier de participer. Ainsi, en 2014, selon la rétrospective officielle de Twitch, les diffusions avaient lieu dans vingt-quatre langues. Ce multilinguisme et cette ampleur internationale rendent compte de l'immense diversité des profils qui se côtoient sur la plateforme.

Bien que dédiée à la diffusion de contenu en lien avec les jeux vidéo, la plateforme mise sur l'aspect interactionnel de ses fonctionnalités, pour se démarquer et créer son identité visuelle. En effet, le slogan, délivré en phylactère rappelle la promesse de Twitch : converser avec les créateurs de contenu. L'aspect original de la plateforme repose sur l'hybridation des différents canaux de communication : sur le canal audio ou vidéo, le joueur diffuse sa partie quand le canal écrit est occupé par les spectateurs. L'intérêt majeur de ce fonctionnement est que les spectateurs 
présents dans l'espace de clavardage interagissent non seulement entre eux mais également avec le joueur durant sa partie.

Ce dernier possède plusieurs canaux de communication. En effet, il est présent à la fois dans l'espace audio-visuel de la diffusion mais également dans l'espace de clavardage. Cependant, l'observation met en évidence une tendance en vigueur sur la plateforme: les joueurs n'interviennent que ponctuellement dans l'espace de clavardage. Celui-ci leur permet par exemple de diffuser des liens, d'annoncer leur venue dans le cadre audio-visuel en début de session, etc. À titre d'illustration, on peut se reporter aux figures 1 et 2, ci-dessous.

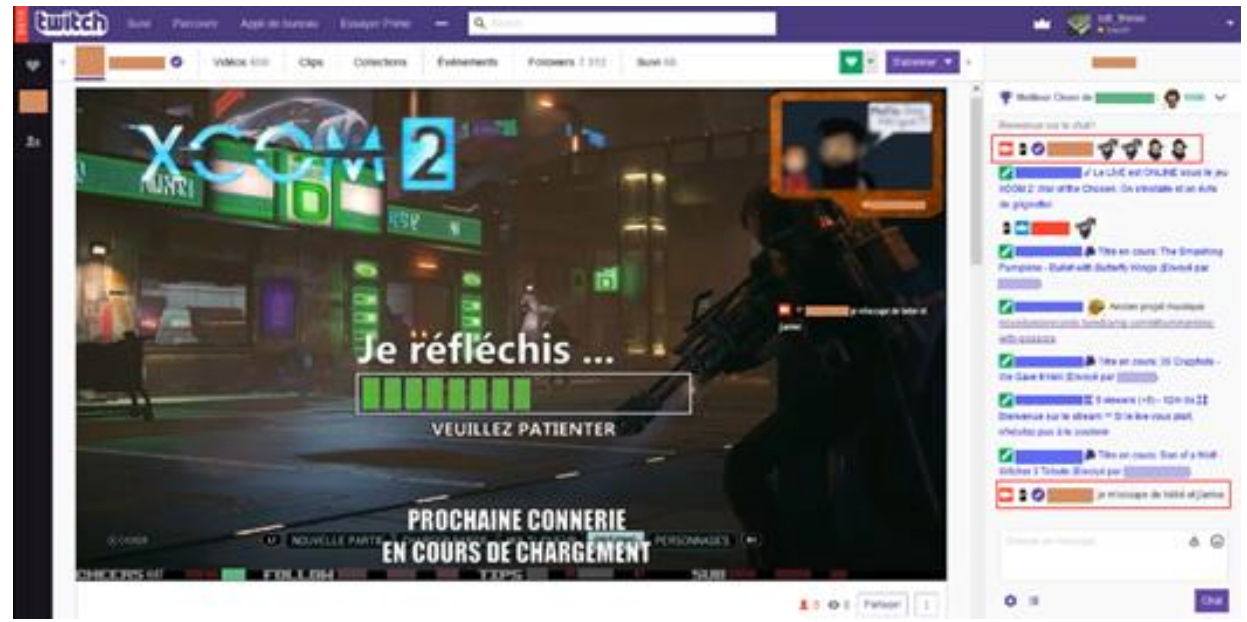

Fig. 1. Session de jeu sur Twitch - 29/09/2017.

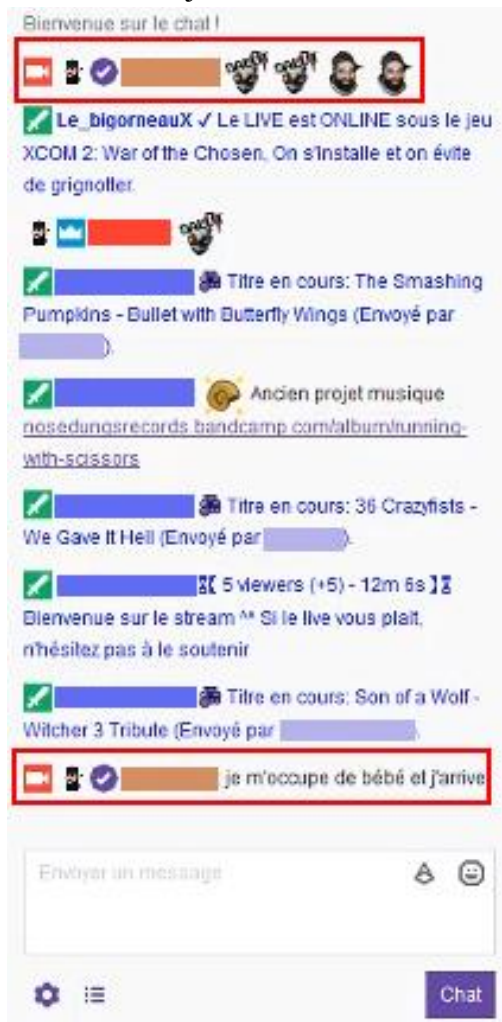

Fig. 2. Chat de session de jeu (détail agrandi de la fig. 1). 
Les énoncés encadrés en rouge dans les photos d'écran ci-dessus renvoient au discours du joueur en début de session. Il n'est pas encore dans l'espace audiovisuel de la diffusion mais il annonce sa venue imminente. Pour cela, il explique ce qui est en train de se jouer dans son espace physique et dévoile un élément intime : il s'occupe de son enfant avant de lancer l'action ludique. Le laps de temps séparant le lancement de la diffusion sur Twitch et celui du jeu vidéo permet aux spectateurs recevant la notification de se connecter sans manquer le début de la session ludique.

Le canal privilégié des joueurs est le canal vocal, accessible au joueur diffusant sa partie. Il peut éventuellement l'ouvrir à une ou plusieurs personnes, le temps d'une partie. Pour cela, il doit passer par un logiciel tiers. En outre, le joueur a la possibilité d'intégrer son image dans le cadre vidéo. Cette configuration, très appréciée des spectateurs, permet à ces derniers d'observer les réactions en direct du joueur.

La spécificité de Twitch tient de cette hybridation entre plusieurs canaux de communication. Contrairement aux plateformes de vidéos à la demande, les producteurs de contenus que sont les joueurs reçoivent des retours au cours de la création. Ainsi, ils peuvent interagir avec leur public pendant leur session de jeu. Cet aspect distingue Twitch des plateformes de diffusion de vidéo en différé (cas de dispositifs comme les chaînes YouTube) et en fait le succès particulier :

«In the case of stream communities, many people watch streams for social interaction with other human beings with whom they identify » (Hamilton, Garretson et Kerne 2014 : 5).

Cette recherche d'interaction rejoint l'idée développée par Ducheneaut (2011: 27) selon laquelle «la sociabilité dans le monde numérique se dirige vers un spectacle social ». Ainsi, Twitch s'offre comme terrain particulièrement propice à l'observation et l'analyse de phénomènes tels que la proximisation discursive et la co-construction de communauté.

\section{Proximité et communauté}

Les échanges dans des contextes numériques sont de plus en plus étudiés par les sciences du langage et, plus largement, par les sciences sociales et humaines ${ }^{\text {ii }}$. Ces disciplines mesurent pleinement de la sorte un enjeu sociétal de notre époque, fortement caractérisée par les dispositifs technologiques voire médiatisée par eux. Néanmoins, l'appréhension de ces fonctionnements suscite de nouveaux défis, surtout relatifs à la multimodalité que présentent les corpus concernés. Cette propriété implique en effet la prise en compte de la complexité contextuelle qu'il convient de décrire minutieusement selon les traditions de l'analyse du discours et de l'ethnométhodologie, en particulier pour ce qu'elle fait émerger de fonctionnements spécifiques, voire singuliers.

L'un de ces fonctionnements, sur lequel notre étude vise à se concentrer davantage, concerne la co-construction de communauté numérique à travers la manifestation et renforcement de la proximité. Rappelons d'emblée que le mot conserve, en roman puis en français, la double valence, physique et abstraite, du latin proximitas « voisinage » ${ }^{\mathrm{iii}}$ et « affinité, ressemblance » (sens figuré). L’idée de proximité, sollicitée de manière croissante ces dernières années, n'obéit pas à une définition établie spécifiquement par une discipline particulière. Le terme n'en est pas moins au centre des multiples réflexions en économie, droit, sciences politiques, géographie ou sociologie ${ }^{\text {iv }}$. On retiendra que ces différentes approches ont en commun de souligner que 
« la proximité renvoie à des relations d'ordre spatial (rapport à un même lieu), tout en pouvant renvoyer à des relations de tout autre type, non spatialisées (au sens de la discontinuité de lieu) »(Bellet et al., 1998, p. 15).

Les auteurs rappellent de plus «que la proximité n'est pas un état, mais un rapport » (Ibid., p. 17).

$\mathrm{Au}$ sein des contextes médiatiques, la proximité est abordée comme faisant partie d'une relation tissée entre l'émetteur et le destinataire, ainsi qu'entre chacun des acteurs et le monde de référence. Pour Fastrez et Meyer (1999), ces relations présentent deux types de proximité : une proximité inscrite et une proximité induite. La première trouve sa place naturellement au sein d'une relation; la seconde correspond à un travail «visant un rapprochement supplémentaire par rapport au stade de la proximité inscrite » (Fastrez \& Meyer, 1999, p. 149) ce que les auteurs appellent « proximisation ». Cette dernière peut avoir lieu avec une nature objective, à travers un rapprochement physique des participants ou de nature subjective, il

« se centre sur la mise en valeur de la proximité existante [...], en visant un accroissement de l'importance de la dimension subjective de la proximité dans le chef d'un ou des deux sujets de la relation, c'est-à-dire une plus grande implication affective du ou des sujet(s) en présence dans la relation » (Ibid., p. 150).

Cette proximisation subjective permet de renforcer la mise en proximité de nos jours où, à travers les réseaux numériques, des personnes de différents endroits peuvent échanger entre elles indépendamment des frontières. Selon Huynen (1997, p. 196),

«il y a donc révolution proxémique dans la mesure où les distances géographiques sans être gommées ne constituent désormais plus l'obstacle majeur à la communication ».

Ainsi, cette procédure favorise l'animation des plateformes numériques qui visent à la constitution d'un groupe de membres, voire à une « communauté ", dont la notion reste étroitement liée à la valeur de base latine d'affinité. Or, même si la notion de proximité est davantage développée dans d'autres disciplines et particulièrement au sein de la sociologie, comme évoqué précédemment, elle est identifiable linguistiquement à travers la verbalisation d'une relation existante ou la valorisation des aspects constitutifs du lien partagé. C'est pourquoi faire appel aux sciences du langage nous semble une porte d'entrée pertinente pour analyser la réalisation discursive de la proximisation au sein des interactions verbales sur Twitch. À cette fin, on mobilisera pour cela principalement la rhétorique, la praxématique et l'analyse discursive des émotions en assurant un lien interdisciplinaire avec l'ethnométhodologie. Précisons que, dans le cadre de notre étude, le terme " communauté » est à prendre au sens resserré de " communities of play» selon Pearce et Artemesia (2011). Partant de la «communauté de pratique » de Wenger (2005) qui désigne les groupes d'apprentissage collectif dans des contextes professionnels, Pearce et Artemesia (2011: 5) proposent ce terme pour distinguer les communautés de joueurs :

« Obviously, communities of practice and communities of play share much in common, and one could even argue that play is a type of practice; however, the adoption of a new term suggests that play practices warrant their own understanding of how communities form and are 
maintained, a subject that becomes particularly pertinent in the context of technologically mediated play $»$.

Dans les communautés de joueurs, ces derniers se reconnaissent, se revendiquent comme tels et développent une véritable identité de groupe, basée notamment sur une appellation comme les «groupes de fan » et diverses marques d'appartenance à cette communauté. Par exemple, les spectateurs de la communauté présentée dans cette étude ont la possibilité de débloquer des émoticônes propres à la chaîne du joueur.

\section{La manifestation discursive du pathos}

La proximité considérée dans notre cadre comme un rapport affectif, social et culturel, se matérialise linguistiquement en faisant appel notamment au pathos, au sens rhétorique du terme, compris comme « les passions projetées sur l'auditoire qui fonderont par suite les linguistes à en lire des marques dans l'énoncé " (Fauré et Perea, 2017 : 120). En effet, il y a des éléments langagiers qui mettent au jour le pathos, tels que les adjectifs qualificatifs, les termes axiologiques, les adverbes et tout type de locution verbale qui projette une modalisation affectivo-subjective ou une forme d'implication des participants dans les propos. Cette projection des passions sur l'auditoire relève bien de la prise en compte des émotions dans le discours. Les émotions, depuis le point de vue sociodiscursif,

« seraient le garant de la cohésion sociale, elles permettraient à l'individu de constituer son sentiment d'appartenance à un groupe (Mauss), elles représenteraient la vitalité de la conscience collective » (Charaudeau, $2000: 2$ ).

Elles font partie du système de représentations et jugements sociaux établi dans un groupe, une communauté ou un peuple. D'après Plantin $(2011: 2)$ par nature, tout discours, même en cherchant à n'être que rationnel et logique, inclut l'aspect émotionnel puisque «représentation rationnelle et émotion sont portées par les mêmes mots, les mêmes constructions, les mêmes arguments ; elles portent aux mêmes intentions de discours ». Cette indissolubilité est essentielle au discours humain, selon Charaudeau (2000:10) :

"Les émotions relèvent d'un état qualitatif d'ordre affectif, du fait d'un sujet qui éprouve et ressent des états euphoriques/dysphoriques en rapport avec sa physiologie et ses pulsions ; mais elle relèvent en même temps d'un état mental intentionnel d'ordre rationnel, en tant qu'elles visent un objet qui est figuré par un sujet qui a une vision sur le monde, qui juge ce monde à travers des valeurs, lesquelles font l'objet d'un consensus social, constituent des savoirs de croyance en imaginaires sociodiscursifs qui servent de support déclencheur à la fois à l'état qualitatif et à une réaction comportementale ».

Cette intentionnalité primaire chez un individu est d'être cru, de convaincre son interlocuteur de la vérité de ce qu'il dit ou de la pertinence de ses propos ou actions. Aristote, dans sa rhétorique, plaçait la relation de complémentarité entre les aspects à caractère logique et ceux d'ordre subjectif : le logos, pour le premier, l'ethos et le pathos, pour les seconds, afin d'atteindre le but persuasif de la communication. Le discours, de même que les énoncés adressés à un spectateur dans le cas des plateformes de diffusion en direct de parties de jeux vidéo, visent en grande partie la persuasion. Cela se justifie par la volonté d'un joueur de se démarquer de la myriade de ses pairs et de fidéliser les spectateurs à sa chaîne. 
En effet, au sein des plateformes numériques, la création et l'animation d'une communauté de pairs deviennent indispensables pour l'existence même desdits sites. Cette communauté se construit à travers des procédures propres à son environnement et c'est pourquoi nous convoquons dans notre étude simultanément la rhétorique et la praxématique, pour laquelle la production de sens ne saurait qu'être articulée au réel. Le contexte, au sens interprétatif, se présente ainsi comme nécessaire à toute compréhension interactionnelle qui l'éclaire en retour. De plus,

« la praxématique rejoint Aristote dans l'importance accordée aux stratégies énonciatives et au travail argumentatif, et dans l'articulation constante $\mathrm{du}$ style et de l'argumentation. Elle enrichit cependant la perspective aristotélicienne en montrant que tout énoncé est un acte de parole en relation avec un point de vue sur le monde qu'on cherche à faire partager à autrui » (Détrie, $2017: 338$ ).

C'est sur cette conception matérialiste et praxéologique que repose notre conception des fonctionnements interprétatifs des échanges, fussent-ils en ligne. Ainsi, l'activité ludique mise en scène (spectacularisée, littéralement) à travers la plateforme et la chaîne vidéo sur Twitch, constitue le réel contextuel qui formate et permet de rendre compte des échanges hybrides qui s'y coproduisent. Dans cette perspective, pour la présente étude, nous allons nous concentrer sur la visée d'adhésion que cherche à transmettre le locuteur principal (le joueur) et qui a comme objectif, implicite ou revendiqué, de susciter le sentiment d'appartenance, de « faire communauté », auprès des spectateurs connectés.

\section{Méthodologie}

La session étudiée est issue d'une série d'enregistrements d'un joueur ayant acquis sur Twitch la possibilité de recevoir des abonnements de la part des spectateurs. Cela veut dire que le joueur diffuse ses sessions de jeu sur un rythme régulier et réunit un certain nombre de spectateurs. L'enregistrement au format vidéo se fait lors de la diffusion en direct grâce au logiciel libre de capture d'écran OBS. Cette méthode permet de capturer à la fois le canal audio, le canal vidéo et le canal écrit (voir figure 1). Les données sont ensuite transcrites et alignées grâce au logiciel d'annotation ELAN. Les transcriptions comprennent à la fois les énoncés oraux et écrits : du joueur, des spectateurs, du bot ${ }^{\mathrm{v}}$ de la chaîne et du jeu en cours. Pour plus de clarté, ne seront reproduits ici que les énoncés pertinents pour notre analyse.

\section{Présentation de la session de jeu}

La session de jeu présentée est issue de la diffusion du 17 octobre 2017 ayant duré $3 \mathrm{~h} 45$ et correspond à la première heure et demie de ladite diffusion. Le jeu en cours s'intitule Le Roi Lion: La formidable aventure de Simba, un jeu de plate-forme édité par Activision et sorti en 2001 sur PlayStation (cf. Fig. 3). Ce jeu a la réputation d'être mauvais et fait régulièrement l'objet de moqueries par les personnes s'intéressant aux mondes vidéoludiques. Si le joueur l'a sélectionné, c'est parce qu'il a pour visée affichée de terminer l'intégralité des 1402 jeux qui composent le catalogue vidéoludique de la Playstation. 


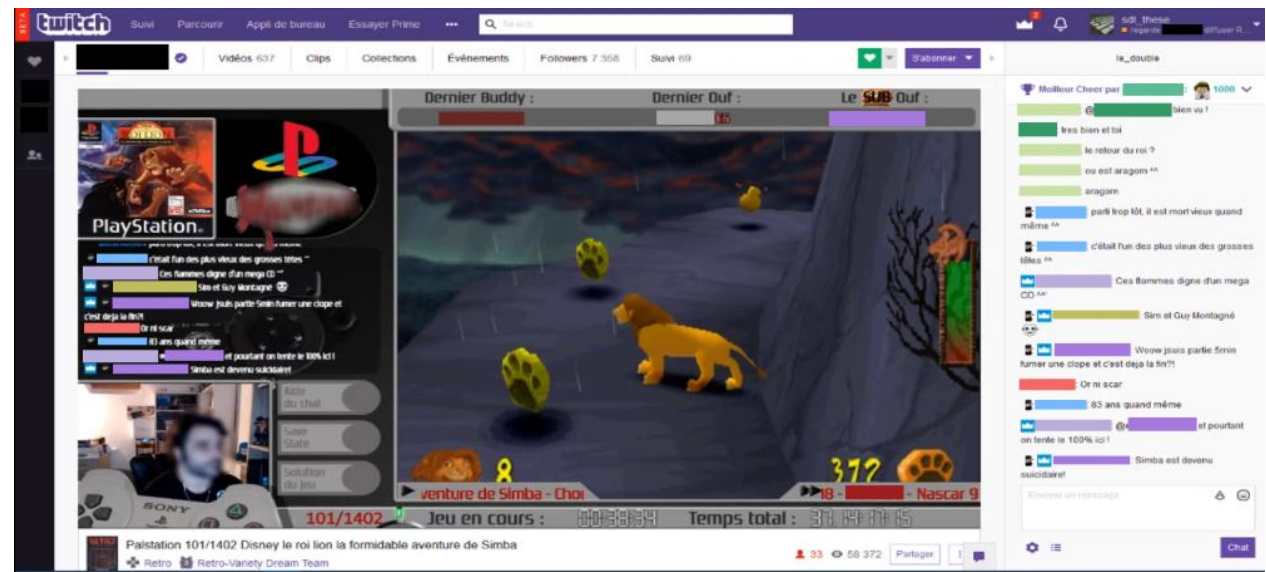

Fig. 3. Photogramme de la session de jeu : « Le roi Lion » sur Twitch $17 / 10 / 2017$.

Sur Twitch, chaque utilisateur enregistré possède une chaîne associée à son profil et peut décider de diffuser ses sessions de jeu. Certaines chaînes regroupent plusieurs joueurs et diffusent divers programmes, un peu comme en télévision. Dans notre étude, le joueur possède sa propre chaîne et est le seul à diffuser sur celle-ci.

Les différents acteurs de la session sont anonymisés comme suit :

- Le joueur, présent à la fois dans le canal audio et vidéo par incrustation de son buste à l'écran du jeu (voir figure 3). Il est identifié comme JOU.

- trente-et-un spectateurs, présents uniquement dans le canal écrit. Ils sont numérotés en fonction de leur apparition dans l'espace de clavardage lors de leur premier message : S01 est le premier spectateur à avoir écrit un message lors de cette session et ainsi de suite jusqu'à S31. Pour information, une des fonctionnalités de Twitch est le compteur de spectateurs en direct. Pour la présente session, il fluctue avec un pic de quarante-six personnes. La différence entre le nombre de spectateurs et le nombre d'énonciateurs sur le canal écrit peut s'expliquer par différents facteurs tels qu'un internaute peut regarder la diffusion même sans compte utilisateur, naviguer sur différentes chaînes à la recherche d'un contenu qui l'intéresse, visionner la session en fond sans participer aux conversations, etc.

\section{Présentation des données}

Durant notre extrait, 1131 énoncés sont produits, dont 900 par le joueur et 431 par les trente-et-un spectateurs. Cet écart s'explique par le fait que le joueur possède une double casquette : en plus de son rôle de joueur, il endosse celui d'animateur de la session. De plus, le canal audio, bien que technologiquement médiatisé, n'est pas aussi contraignant que l'utilisation d'un clavier pour s'exprimer dans le canal écrit.

\section{Analyse}

Faisant un bref état des lieux de la littérature sur la question de la socialisation des joueurs dans les jeux vidéo en ligne, Rueff (2011) a remarqué la récurrence de l'« envie de socialiser » comme motivation principale pour les joueurs. Sur Twitch, 
cette tendance semble ressortir d'autant plus qu'un seul utilisateur joue. Dès lors, l'activité ludique semble n'être qu'un prétexte à une réunion conversationnelle pour les sujets engagés dans les interactions. Ce terreau propice à la proximisation permet d'observer des éléments discursifs participant de la co-construction de communauté. Nous insisterons à ce titre sur la manifestation dans notre corpus de trois catégories d'observables affiliatifs - au sens interactionnel et en termes de moralité pratique des participants à un échange, de production d'attitudes convergentes (stances, selon Stivers, 2008)-: les salutations, les engagements pris devant la communauté, l'enregistrement en direct d'un nouvel abonnement à la chaîne. Leur propriété commune est d'instancier des fonctionnements globaux (macrostructurel, à portée sociale) par des formes et énoncés locaux (de microstructuration).

\section{Les salutations}

La salutation constitue le tour d'ouverture de tout échange communicatif : grâce à lui, le premier interactant place "l'axe de formalité » (Kerbrat-Orecchioni, 1990 : 127). Dans notre cas d'étude, le joueur-animateur (JOU) ouvre la diffusion et détermine ce degré en « familier » voire en « très familier », en l'occurrence, sous la formulation de premier tour : «bonsoir bonsoir et bienvenue ». Après un peu plus de cinq secondes, JOU répète la salutation et en réitère ensuite l'acte de langage $a$ posteriori, de manière personnalisée auprès de chaque internaute connecté avec "salut», suivi du pseudonyme du spectateur. Ce pseudonyme n'est pas intégralement énoncé par JOU, qui, en tendance, le tronque ou lui adjoint un suffixe hypocoristique «-où ». Cette attribution d'un surnom renforce la proximité entre lui, en tant que joueur-animateur et instance de production, et les spectateurs, instance de réception non passive. Sont ainsi exhibées à la fois une mise en confiance mais aussi une valorisation de chacun puisque, même si le spectateur se connecte une fois la partie commencée, JOU va le saluer sans interruption du jeu en cours. Cette procédure relève du travail de proximisation effectué par JOU dans son intérêt, qui est de mettre en avant le lien existant entre eux, la valorisation individuelle permettant à chaque spectateur de se sentir pris en considération et affiliativement intégré à la co-construction de la partie en cours. Par ailleurs, le "salut» utilisé généralement peut alterner avec une salutation encore plus familière « yo [pseudonyme tronqué] bien », ce qui accentue l'informalité de l'échange et la mise en avant de la relation de confiance propre à une communauté, dont les membres peuvent communiquer entre eux, de manière spontanée et à égalité de droits et de devoirs interactionnels, selon les registres de la conversation familière et ordinaire en français.

\section{Extrait 1 : Respecter ses engagements}

Nous allons concentrer notre analyse sur un échange en particulier qui se tient après 8 minutes 48 secondes de jeu. Ce dernier n'a d'ailleurs pas commencé immédiatement au lancement de la diffusion, qui a débuté environ vingt minutes avant l'extrait retranscrit suivant ${ }^{\mathrm{vi}}$ :

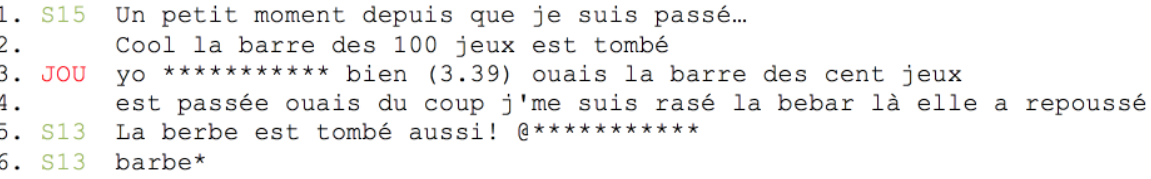

Retranscription de l'extrait 1 (clavardage de la partie en cours). 
S15 commence son tour en manifestant sa satisfaction de voir un objectif atteint durant son absence aux dernières parties : «la barre des 100 jeux est tombé ». Ce dernier énoncé est introduit par un modalisateur avec l'adjectif évaluatif à valeur adverbiale «cool». Cette marque est caractéristique du registre familier, établi, comme on l'a vu supra dès la séquence d'ouverture de chacune des parties de JOU. $\mathrm{Au}$ tour initiatif de S15, qui n'inclut pas de marque manifeste de salutation, la réponse de JOU passe par son propre salut rituel. Il encadre ensuite de l'interjection familière de confirmation « ouais » une première proposition résomptive : <la barre des cent jeux est passée $>$, en indiquant la conséquence induite par le contenu propositionnel de cette dernière : "j'me suis rasé la be-bar», qui implicite un engagement perlocutoire antérieur pris par JOU pour le moment où il atteindrait la quantité de jeux précitée. Dans cette séquence narrative rétrospective, l'emploi en connecteur logique de « du coup » qui articule les deux propositions, met en relief la relation de cause-conséquence entre les deux unités de construction de tour. Elle constitue aussi, par ricochet, un rappel de la tenue de l'acte promissif implicité pour S13 mais aussi pour le tiers symbolisant que forme le public connecté. C'est dans ce cadre que, toujours à propos de ladite barbe, l'incise autojustificatrice : " là elle a repoussé » vise à éclairer S15 qui n'a pu assister à plusieurs des sessions précédentes. L'intégralité du tour de JOU revêt de la sorte une double valence affiliative : d'une part, il offre un accueil personnalisé à l'un de ses supporteurs et, de l'autre, il souligne le respect de ses engagements, inscrivant dans la durée de la chaîne sur Twitch la séquentialité de ses actions et de ses propos. Le commentaire de S13 (en tour 5) vii confirme la visée affiliative, par un membre de la communauté, à travers l'espace de clavardage. Le décalage technologique et la différence des canaux (écrit et sonore) empêche d'établir si S13 émet sa contribution pour attirer l'attention de S15 sur le fait que JOU s'était rasé ou s'il l'énonce en réponse au tour de JOU. Néanmoins, cet échange atteste de l'importance accordée par les deux types d'acteurs, joueur et spectateurs, aux accords établis entre eux. Ainsi, l'agir de JOU manifeste une fois de plus un travail de proximisation à dominante subjective (selon les termes de Fastrez et Meyer, 1999) réalisée à travers une mise en exergue de l'accomplissement des accords. Cela peut montrer le respect qu'il a pour ses spectateurs, la crédibilité qu'il recherche à travers la tenue de ses engagements : du rapport de confiance ainsi obtenue des spectateurs envers lui, doit découler leur fidélisation.

\section{Extrait 2 : Réagir à un abonnement}

L'échange suivant survient quarante-huit minutes après le début du jeu et dure un peu moins de cinq minutes. Pour plus de clarté dans la compréhension de l'échange, seuls les énoncés des deux interlocuteurs principaux et du bot sont ici reproduits.

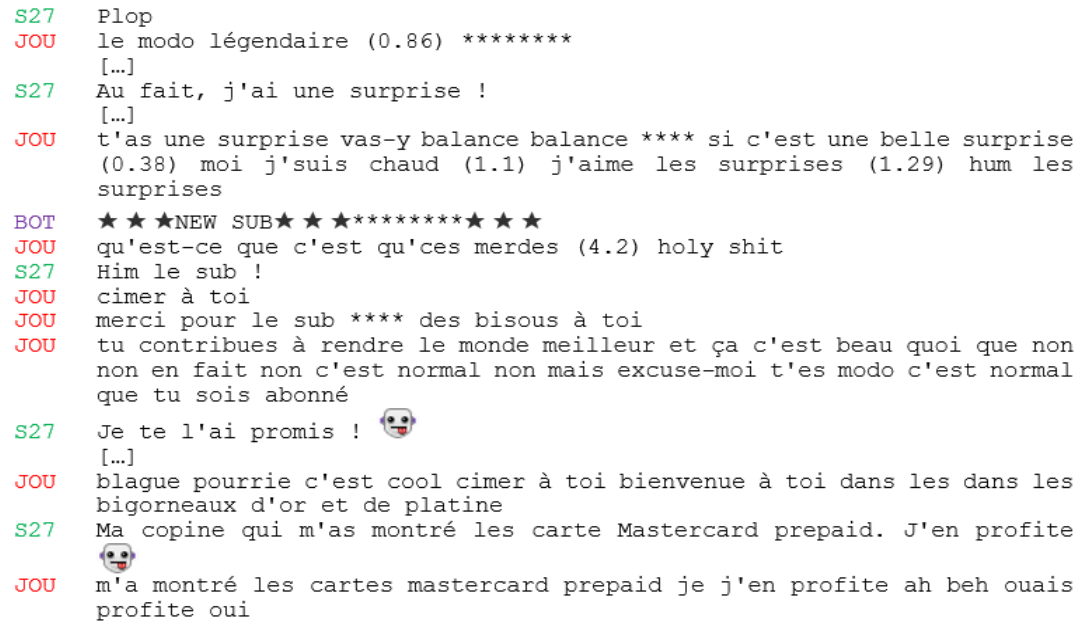


Transcription de l'extrait 2 (clavardage de la partie en cours).

Les deux segments constitués de huit astérisques en tours 2 et 13 correspondent au pseudonyme anonymisé de S27. Contrairement aux autres spectateurs, ce dernier n'est pas salué par le joueur. En effet, le tour 2 est constitué d'un groupe nominal puis du pseudonyme de S27. Le groupe nominal «le modo légendaire » caractérise S27 en mettant en avant son rôle de modérateur sur la chaîne. Ce statut (non exclusif: d'autres modérateurs participant à la session) lui a été conféré par le joueur et lui donne l'autorité dans l'espace de clavardage : il peut bannir un spectateur, imposer qu'un laps de temps s'écoule entre deux messages d'un même énonciateur, etc. L'emploi marqué de l'adjectif " légendaire » souligne la proximité relationnelle entre les deux interlocuteurs et la place de S27 au sein de la communauté, dont il apparaît parfaitement connu. En tour 4, S27 annonce avoir une surprise sans en préciser le destinataire. Pourtant, JOU répond en encourageant le dévoilement de ladite surprise par l'emploi de plusieurs impératifs « vas-y balance balance » suivi du pseudonyme tronqué de S27. Le surnom marque une fois de plus la proximité entre les deux interlocuteurs. Quant aux impératifs, ils marquent l'impatience de JOU face à l'annonce de S27. On notera qu'au cours de la diffusion, c'est JOU qui, en principe, crée l'attente des spectateurs vis-à-vis de son contenu. Ici, les rôles s'inversent et S27 prend le pouvoir jusqu'à la révélation de sa surprise - son abonnement payant à la chaîne de JOU - qui apparaît par l'intervention du bot en tour 9 et un évènement visuel dans l'interface du joueur. En effet, lorsqu'un spectateur s'abonne, une musique caractéristique ${ }^{\text {viii }}$ se déclenche et un gifix de Crash Bandicoot $t^{x}$ apparaît dans le canal vidéo, assorti du message : «******** a fauté, danse maintenant ». JOU se met alors à danser entre le tour 10 après le juron « holy shit » et le tour 12. Cette danse s'ensuit de remerciements (tours 12 et 13) et du gimmick $^{\mathrm{xi}}$ de JOU en tour 14 : « tu contribues à rendre le monde meilleur et ça c'est beau ». Ce gimmick est prononcé par JOU à chaque fois qu'un spectateur s'abonne ou suit sa chaîne. Il adopte alors une intonation et une hauteur de voix différentes du reste de ses productions discursives. Sa mimogestualité change également: il bascule sa tête de manière à être de trois-quart face à la caméra, hausse un sourcil et affiche un sourire toutes dents affichées. Cependant, dans notre échange, ce gimmick est immédiatement complété par un retour à son attitude normale et d'un énoncé le contrebalançant: « quoi que non non en fait non c'est normal non mais excuse-moi t'es modo c'est normal que tu sois abonné ». Cet énoncé a une visée humoristique qui joue sur le statut de S27 précédemment décrit. Toujours dans l'esprit de l'expression d'un humour affiliatif, au tour 20, JOU emploie le terme «bigorneaux ». Il s'agit pour cette communauté d'une marque d'appartenance emblématique mais autodérisoire. Elle n'est pas seulement marque linguistique puisque les spectateurs utilisent régulièrement une émoticône en forme de coquillage. Bien que cela n'apparaisse pas dans l'échange, l'un des messages programmés du bot est: « N'hésitez pas à lancer des bigorneaux quand le streameur est impressionnant et/ou scandaleux » et JOU y fait régulièrement allusion. Enfin, l'échange se termine par un phénomène récurrent dans les interactions se produisant sur Twitch: la lecture à haute voix par le joueur d'un énoncé produit par le spectateur. Au tour 23, JOU reprend en partie le tour 21, produit par S27. Cette attitude lui permet de marquer la reconnaissance et la prise en compte de S27. Il n'est pas toujours aisé pour un joueur de procéder de la sorte car, pour lire les énoncés de l'espace de clavardage, il doit quitter l'écran de jeu des yeux, ce qui est incompatible avec la pratique du jeu vidéo. Les joueurs développent différentes techniques pour contourner cette difficulté : mettre le jeu en pause et lire plusieurs énoncés d'un coup, attendre une cinématique ou un chargement entre deux 
niveaux, etc. Dans notre extrait, JOU profite de la mort de son avatar. Cette lecture est suivie d'un commentaire de JOU « ah beh ouais profite oui ». Par l'emploi de l'impératif du verbe transitif « profiter », des mots-phrases « oui » et « ouais », JOU approuve l'énoncé de S27 et l'encourage. Le tutoiement n'est ici qu'un indice du faisceau de données contextualisant les énoncés de proximisation et de familiarité au sein de la communauté.

L'utilisation par le joueur et les spectateurs de canaux de communication différents n'appauvrit pas les échanges proprement linguistiques. En effet, l'hybridation entre écrit et oral réactualise les pratiques et les interactions. Le joueur, concentré dans sa partie, lit et prend en compte les énoncés écrits dès qu'il le souhaite ou peut. Les spectateurs, bien qu'ils puissent mener plusieurs activités en parallèle dans leur propre espace physique ou numérique (ouverture de plusieurs onglets par exemple) n'éprouvent pas a priori la charge mentale que représente l'action ludique. Cela leur permet de prendre le temps de rédiger des messages écrits. Ainsi, pour le joueur, il est avantageux d'occuper le canal vocal : il peut produire des énoncés tout en gardant l'appareillage technologique (manette, clavier, souris, etc.) nécessaire à son jeu en main. De plus, la spontanéité de l'oral est très appréciée des spectateurs. Enfin, bien que les spectateurs développent des conversations entre eux, ils témoignent d'une envie de construire des échanges avec le joueur. Cette tendance repose sur les limites du clavardage qui peut voir se multiplier les interventions au risque d'en lasser les lecteurs :

«However, as streams scale up, information overload renders chat unreadable, and moderation becomes overwhelming. [...] However, participants become frustrated with the difficulty of interacting in these streams. We found that for this reason, many choose to participate in smaller streams, which they experience as affording more meaningful interaction » (Hamilton, Garretson et Kerne, 2014).

Ainsi, l'utilisation du canal oral par le joueur lui permet de réagir, en lecture, à plus d'énoncés de l'espace de clavardage que s'il devait passer par le canal écrit. En effet, la vitesse de production réduite de l'oralité par rapport à l'écrit apparaît ici comme un avantage majeur dans l'animation d'une session de jeu à destination de spectateurs encouragés à interagir.

\section{Conclusion}

La pratique du jeu vidéo devant des spectateurs n'est pas nouvelle - pensons aux parties dans un salon devant des ami.e.s ou des membres de la famille. Ce que change Twitch, c'est le non-partage d'un même espace physique et d'une pratique commune simultanée d'un jeu: chaque internaute possède son espace, sans participation ludique active. Le cadre commun est en revanche l'espace médiatique. Les spectateurs sont alors des inconnus mus par une même passion vidéoludique et les joueurs sont nombreux à proposer des diffusions de contenu. Cette reconfiguration d'une pratique déjà existante $\mathrm{a}$, entre autres, pour conséquence de faire porter aux joueurs un rôle d'animateur. Ils doivent se démarquer et s'ils jouent sur une plateforme telle que Twitch, c'est bien par volonté de partage et d'interaction avec les autres. Dès lors, la construction et l'entretien d'une communauté, aussi petite soit-elle, constitue un atout majeur pour ces joueurs/animateurs. 
Qu'il s'agisse de formes nominales d'adresses complétées ou non par des marques d'affection, de prise en compte de l'autre, de respect des engagements pris ou encore d'échanges à caractère humoristique, toutes les formes de reconnaissance de l'instance réceptrice (les spectateurs) par l'instance productive (le joueur) permettent de créer une proximité relationnelle entre les interactants. De plus, le spectateur n'est pas un récepteur passif puisqu'il peut produire des énoncés tout au long de la diffusion de la partie et devient par là un créateur de contenu au même titre que le joueur. Les deux instances sont complémentaires et la richesse de leurs échanges est un terreau fertile pour l'étude de ces phénomènes. Toutes ces marques participent de la co-construction d'une communauté dont les membres partagent non seulement des centres d'intérêt, des pratiques mais également un historique.

Enfin, la relation entre le joueur et les spectateurs dépasse bien souvent le cadre de Twitch par un prolongement de leurs échanges sur d'autres réseaux tels que Facebook, Twitter, Discord, etc. Dans le cas étudié, le joueur a créé un document en ligne accessible à tous, qui liste les différents jeux de la PlayStation. Dès qu'il en termine un, il consigne seulement la date de l'accomplissement, le temps de jeu et autres informations liées à l'action de jouer mais également le pseudonyme de l'internaute le lui ayant proposé. Ainsi, l'implication de ce dernier est reconnue, même a posteriori. La proximisation relationnelle est alors marquée et perdure dans le temps.

\section{Bibliographie}

Bellet, M., Kirat, T., \& Largeron, C. (1998). Approches multiformes de la proximité. Paris : Hermès.

Charaudeau, P. (2000). La pathémisation à la télévision comme stratégie d'authenticité. Dans C. Plantin, M. Doury et V. Traverso (dirs.), Les émotions dans les interactions. Lyon : Presses universitaires de Lyon.

Denouël, J. (2008). La notion de «tour» dans l'analyse des interactions médiatisées par ordinateur. Cahiers de praxématique, [En ligne] ,50 | mis en ligne le 01 janvier 2013.

Garcia A. C. et Jacobs J. B. (1999). The Eyes of the Beholder: Understanding the TurnTaking System in Quasi-Synchronous Computer-Mediated Communication, Research on Language and Social Interaction, 32: 4, p. 337-367.

Mattio, V. (2004). Les ressources sûres des cyberconversations. Analyse goffmanienne des interactions sur le dialogue en direct de Caramail, COMMposite, v2004.

Rheingold, H. (1993). Chapter Six: Real-time Tribes, in Rheingold, H. The Virtual Community, version en ligne : http://www.rheingold.com/vc/book/6.html.

Bourdieu, P. (2014). Les structures sociales de l'économie. Paris : Éditions du Seuil.

Chabot, G. (1954). L'armature urbaine en géographie régionale. In: Urbanisme et architecture. études écrites et publiées en l'honneur de Pierre Lavedan. Poitiers : Cahiers de Civilisation Médiévale.

Goffman, E. (2002). L'arrangement des sexes. Paris : La Dispute.

Grossetti M. (2004). Sociologie de l'imprévisible. Dynamiques de l'activité et des formes sociales. Paris : PUF. 
Kirat, T., Lung, Y. (1995). Innovations et proximités : le territoire, lieu de déploiement des processus d'apprentissage. In Coordination économique et apprentissage des firmes, (Eds, Lazaric N., Monnier J. M.), Paris : Economica.

Sorokin, P. A. (1959). Social and Cultural Mobility. Containing complete reprints of Social Mobility and Chapter V from Volume IV of Social and Cultural Dynamics. Londres: Collier-Macmillan Ltd.

Detrie, C. (2017). Rhétorique. Dans C. Détrie, P. Siblot, B. Verine et A. Steuckardt (éds.), Termes et concepts pour l'analyse du discours. Une approche praxématique (p. 337-339). Paris : Honoré Champion Éditeur.

Ducheneaut, N. (2011). Les jeux vidéo en ligne : un laboratoire virtuel de recherche en sciences sociales ? Dans C. Perraton, M. Fusaro et M. Bonenfant (dirs.), Socialisation et communication dans les jeux vidéo. Québec : Les Presses de l’Université de Montréal.

Fastrez, P., \& Meyer, S. (1999). Télévision locale et proximité. Recherches en communication, (11), 143-167.

Fauré, L. et Perea, F. (2017). Émotion. Dans C. Détrie, P. Siblot, B. Verine et A. Steuckardt (ed.), Termes et concepts pour l'analyse du discours. Une approche praxématique (p. 117-122). Paris : Honoré Champion Editeur.

Hamilton W. A., Garretson O., et Kerne A. (2014). Streaming on Twitch; Fostering Participatory Communities of Play within Live Mixed Media. Dans M. Jones et P. Palanque (dirs.), CHI'14 Proceedings of the SIGCHI Conference on Human Factors in Computing Systems. New York : ACM.

Huynen, C. (1997). La proximité dans l'esprit du temps. Recherches en Communication, 7. https://doi.org/10.14428/rec.v7i7.46493

Kerbrat-Orecchioni, C. (1990). Les interactions verbales. Paris : A. Colin.

Pearce, C. et Artemesia, (2011). Communities of plays: emergent cultures in multiplayer games and virtual world. Cambridge : The MIT Press.

Plantin, C. (2011). Les bonnes raisons des émotions. Principes et méthode pour l'étude du discours émotionné. Berne : Peter Lang.

Rueff, J. (2011). Formes de reconnaissance et de mépris dans les mondes numériques : une étude des valorisations sociales à l'oeuvre dans les interactions médiatisées des joueurs de Warhammer online. Travail de thèse soutenu à l'UQAM disponible en ligne : https://archipel.uqam.ca/4965/1/D2305.pdf

Stivers, T. (2008). Stance, alignment and affiliation during storytelling: when nodding is a token of affiliation, Research on Language and Social Interaction, vol. 41, $\mathrm{n}^{\circ} 1,31-57$.

Wenger, E. (2005). La théorie des communautés de pratique. Apprentissage, sens et identité. Saint-Nicolas, Québec : Presses de l'Université Laval.

\footnotetext{
i Voir à ce propos le rapport annuel sur l'état des plateformes de streaming de StreamElements et Arsenal (https://cdn.streamelements.com/static/State-of-Stream-2019.pdf)

ii Citons par exemple Denouël (2008) ; Garcia et Jacobs (1999) ; Mattio (2004); Rheingold, H. (1993).

iii Il est dérivé de proximus (« le plus proche ») et du superlatif de propior («proche »).
} 
iv Bourdieu, P. (2014) ; Chabot, G. (1954) ; Goffman, E. (2002) ; Grossetti M. (2004) ; Kirat, T., Lung, Y. (1995) ; Sorokin, P. A. (1959).

$\checkmark$ Le bot correspond ici à un programme informatique ayant plusieurs fonctions au sein de l'espace de clavardage, notamment la régulation des messages comportant des mots interdits, la diffusion de messages pré-enregistrés renvoyant vers les divers réseaux sociaux du joueur, etc.

vi Les énoncés des spectateurs étant écrits, ils sont ici retransmis tels quels sans modification orthographique. Les interlocuteurs s'exprimant à travers l'espace de clavardage sont identifiés par la couleur verte. Le joueur est spécifié par la couleur rouge. Le bot du joueur l'est par la couleur mauve.

vii L'autocorrection de la faute de frappe est assortie de l'astérisque méta-évaluatif suffixé au mot, qui vaut pour instruction <annule et remplace> dans les échanges numériques pour préciser que c'est une correction et pas un ajout.

viii Il s'agit de Super Freak, de Rick James.

${ }^{i x}$ C'est-à-dire une image animée.

x Sage de jeux de plateformes dont le premier opus est sorti en 1996 sur la Playstation et dont le héros est devenu emblématique

xi Énoncé récurrent qui permet l'identification de son auteur. 